\title{
Renal Bioengineering with Scaffolds Generated from Rat and Pig Kidneys
}

\author{
Marina Figliuzzi ${ }^{a} \quad$ Giuseppe Remuzzi ${ }^{a}$ b $\quad$ Andrea Remuzzia, c \\ a IRCCS - Istituto di Ricerche Farmacologiche 'Mario Negri', Centro Anna Maria Astori, Science and Technology Park \\ Kilometro Rosso, Bergamo, ${ }^{b}$ Unit of Nephrology and Dialysis, Ospedale Giovanni XXIII, Bergamo, and ${ }^{\mathrm{C}}$ Department \\ of Industrial Engineering, University of Bergamo, Dalmine, Italy
}

\section{Key Words}

Regeneration · Scaffolds · Chronic kidney disease

\begin{abstract}
Background: Chronic kidney disease (CKD) is a global public health issue with an estimated prevalence of $8-16 \%$ worldwide. End-stage renal disease eventually develops every year in $0.15-0.2 \%$ of patients with overt CKD, and renal replacement therapy (RRT) with dialysis or transplantation is required. Although approximately 2 million people worldwide are currently on RRT to sustain life, this likely represents less than $10 \%$ of those who need it. The kidney transplant approach is also seriously impaired by limited graft survival and by the scarce availability of donors. Innovative tissueengineering strategies have been recently proposed to overcome these challenges. It is anticipated that these novel approaches will also be cost-effective in the long term. Although the initial setup of these innovative technologies could be quite expensive, there would be a single application for each patient, with no additional costs thereafter, compared to the lifelong costs of dialysis or immunosuppressive medications required for transplantation. One of the most innovative tools currently being investigated in experimental models is based on the idea of using decellular-
\end{abstract}

ized kidneys to engineer a new functional organ as a potential future treatment option for end-stage renal disease. Summary: In the last 5 years, several interesting observations have been reported regarding the possibility of using an acellular matrix from the whole kidney and the attempt to recellularize this scaffold using stem or differentiated cells. This review provides an overview of the decellularization methods tested so far and their effects on the resulting extracellular matrix structure and composition. In addition, we also discuss methods recently described by us and others for the perfusion of kidney scaffolds for recellularization. Key Messages: Despite difficulties in achieving the import goal of kidney engineering in the laboratory, we discuss the problems with and limits of the experimental results obtained so far and point out the strategies that need to be adopted in order for this line of research to advance.

@ 2014 S. Karger AG, Basel

\section{Introduction}

Chronic renal disease (CKD) is a progressive condition marked by the deterioration of kidney function over time. Eventually, kidney failure ensues and renal replacement therapy (RRT) is required. The Global Burden of

\section{KARGER}

E-Mail karger@karger.com

www.karger.com/nee (c) 2014 S. Karger AG, Basel

$1660-2129 / 14 / 1262-0113 \$ 39.50 / 0$
Andrea Remuzzi, EngD

Department of Biomedical Engineering, IRCCS - Istituto di Ricerche Farmacologich 'Mario Negri', Centro Anna Maria Astori, Science and Technology Park Kilometro Rosso Via Stezzano 87, IT-24126 Bergamo (Italy)

E-Mail andrea.remuzzi@marionegri.it 
Diseases 2010 study reported that CKD contributes to approximately 740,000 deaths annually, making it the 18 th leading cause of death [1]. Approximately 2 million people are currently being treated with RRT, which consists primarily of dialysis and kidney transplantation. More than $90 \%$ of these individuals live in industrialized countries, while the availability of RRT in developing countries is scarce and it is null in very-low-resource regions, resulting in over 1 million deaths annually from untreated kidney failure [2]. Treatment of even a relatively limited number of patients represents a major societal commitment since RRT absorbs a significant proportion of the health care budget. Furthermore, patient outcomes in dialysis are disappointing, with deaths from accelerated cardiac disease and infections contributing to an annual mortality rate exceeding $25 \%$. Successful renal transplantation improves the quality of life and increases survival compared to long-term dialysis treatment [3]. Nevertheless, because of the limited organ supply and the increasing demand, many patients who need transplants do not receive them and die while on the waiting list. Thus, a severe shortage of cadaveric organ donors is currently the major hurdle to overcome in order to meet the needs of transplant programs, eventually limiting the number of patients who benefit from this therapy worldwide.

Innovative bioengineering strategies that would help to simultaneously overcome the challenges of reduced access to dialysis and the need for organs for transplantation have been recently proposed. The focus is on the idea of generating new kidneys via tissue engineering technologies starting from a whole-kidney scaffold with an intact 3-dimensional (3D) geometry and vasculature obtained by decellularization of a kidney harvested from the patient with CKD. Recellularization of the scaffold with patient-specific progenitor cells would eventually lead to the generation of a new transplantable organ. This review summarizes the most promising results achieved so far in the field of kidney tissue engineering and discusses the hurdles that need to be overcome in order to make this innovative strategy a promising future clinical tool for CKD patients.

\section{Kidney Scaffold Production by Decellularization Procedures}

Biological scaffolds composed of extracellular matrix (ECM) are commonly used for a variety of reconstructive surgical applications and they are increasingly being used in regenerative medicine for tissue and organ replacement [4]. Biological scaffolds are mainly composed of ECM and are usually obtained by decellularization of tissues or organs. Organ ECM is composed of different organizations of complex interconnected fibrous networks of structural and functional molecules including mainly proteins, glycoproteins, carbohydrates, and growth factors. The ECM represents the secreted products of the resident cells of each tissue and organ and is in a state of dynamic equilibrium with these cells and the surrounding environment [5]. The ECM provides structural support for cells that reside on the matrix, with specific mechanical properties, such as rigidity or elasticity, providing bioactive cues for the regulation of cell activities, acting as a reservoir of growth factors, and providing a dynamic degradable environment to allow tissue healing and remodeling. The ECM possesses the ideal characteristics of a tissue-engineering scaffold, i.e. it is a biocompatible, supportive medium for blood vessels, nerves, and lymphatics and for the diffusion of nutrients from the bloodstream $[6,7]$.

The advantage of using acellular organ scaffolds is that it is possible to use ECM not only for structural functions but especially for signals that can be exchanged with adhering cells to induce their migration and differentiation $[5,8]$. These features are very important when complex tissues with different cellular components are involved. The native composition and the macroscopic structure of ECM scaffolds can be preserved using appropriate processing steps for decellularization of the tissue and organs $[5,9]$. The complex anatomy and physiology of the kidney make decellularization more complex, compared to other organs, because of the high number of differentiated cells and its complex micro- and macrostructure.

One of the first reports in the literature of kidney decellularization is that of Ross et al. [10]. The protocol of decellularization used in that study was based on continuous washing of rat kidneys for 5 days through the renal artery by perfusion of ionic and nonionic detergents, and enzymatic degradation of the cellular nuclear material. Nonionic detergents, however, may be traumatic to the tissue and may disrupt the collagen structure. More recent reports available in the literature [10] have shown that whole-organ scaffold, with an intact 3D geometry and vasculature, can be obtained by perfusing rat kidneys with an anionic detergent such as sodium dodecyl sulfate (SDS). In our laboratory, after testing several decellularization strategies, we currently use only SDS as a detergent, followed by rinsing with hypotonic distilled water to completely eliminate the detergent. We obtained com- 
plete cell removal within $17 \mathrm{~h}$ by rapid infusion of SDS detergent into the artery of rat kidneys under physiological pressure, as shown in figure $1 \mathrm{a}, \mathrm{b}$. The decellularized scaffolds preserved the 3D architecture of the glomeruli and tubuli, as shown by scanning electron microscopy analysis (fig. 2), and the integrity and patency of the vascular network (fig. 3). With this protocol we verified, via immunostaining of the ECM, the presence of type IV collagen, laminin, and fibronectin, similarly to the expression patterns of these proteins in native kidney tissue [unpubl. data]. Other groups obtained similar results in rat kidney decellularization and produced well-preserved acellular matrices using detergent perfusion into the renal artery $[11,12]$.

Recently, kidney scaffolds consisting of acellular matrices have been successfully obtained also from pig kidneys [13]. These scaffolds have retained the architecture of the native kidney and, in particular, of the vascular tree both on the arterial side and on the venous side. In this report, a decellularized kidney was transplanted into a pig, even without recellularization, to verify the possibility of connecting the acellular matrix to the vasculature of the recipient and to assess whether blood circulation could be effectively restored. Even in the absence of cells within the matrix, it was possible to demonstrate adequate blood perfusion with constant blood pressure. As expected, however, within minutes a massive thrombosis took place with the presence of inflammatory cells and thrombi within the acellular matrix. More details on the application of the decellularization process in the human kidney are reported in the next section.

The ultimate goal of any decellularization protocol is to remove all cellular material without adversely affecting the ECM composition, biological activity, and mechanical integrity. The detergents used for organ decellularization have been shown to potentially disrupt the collagen of certain tissues $[14,15]$. Moreover, the molecular composition of the native ECM scaffold structure is critical to direct the cell seeding and reconstitution of individual cellular compartments. Sullivan et al. [13] showed that decellularized porcine kidney with SDS retained an intact microarchitecture, including the renal microvasculature. Histological and quantitative analyses of ECM components showed the preservation of glycosaminoglycans and collagens. Nakayama et al. [16] demonstrated that SDS was most effective at preserving the native architecture of the rhesus monkey kidney. Immunohistochemistry demonstrated the expression of common ECM proteins, including heparan sulfate proteoglycans, collagens, and laminin, in decellularized scaffolds. All of these ECM
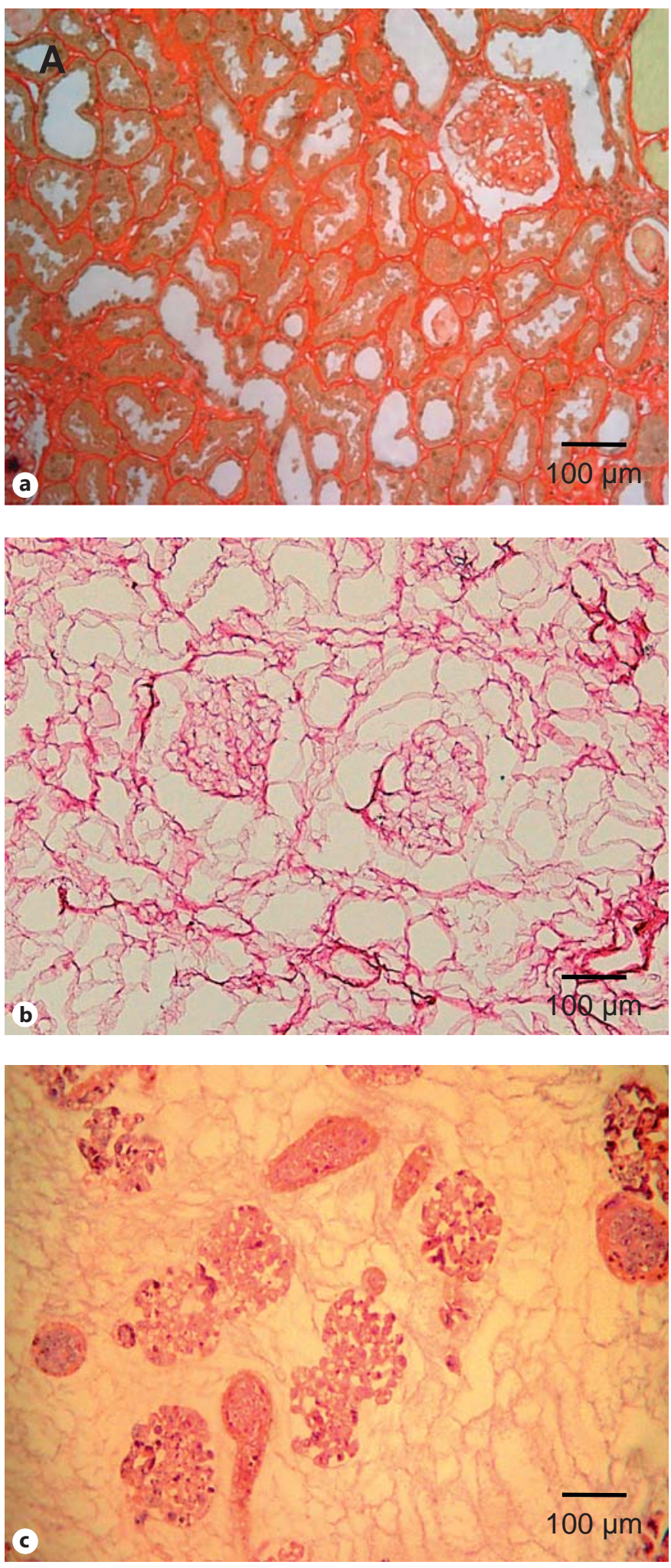

Fig. 1. Sirius red staining of a control kidney (a) and HE staining of decellularized (b) and recellularized kidneys (c) (original magnification $\times 20$ ). 

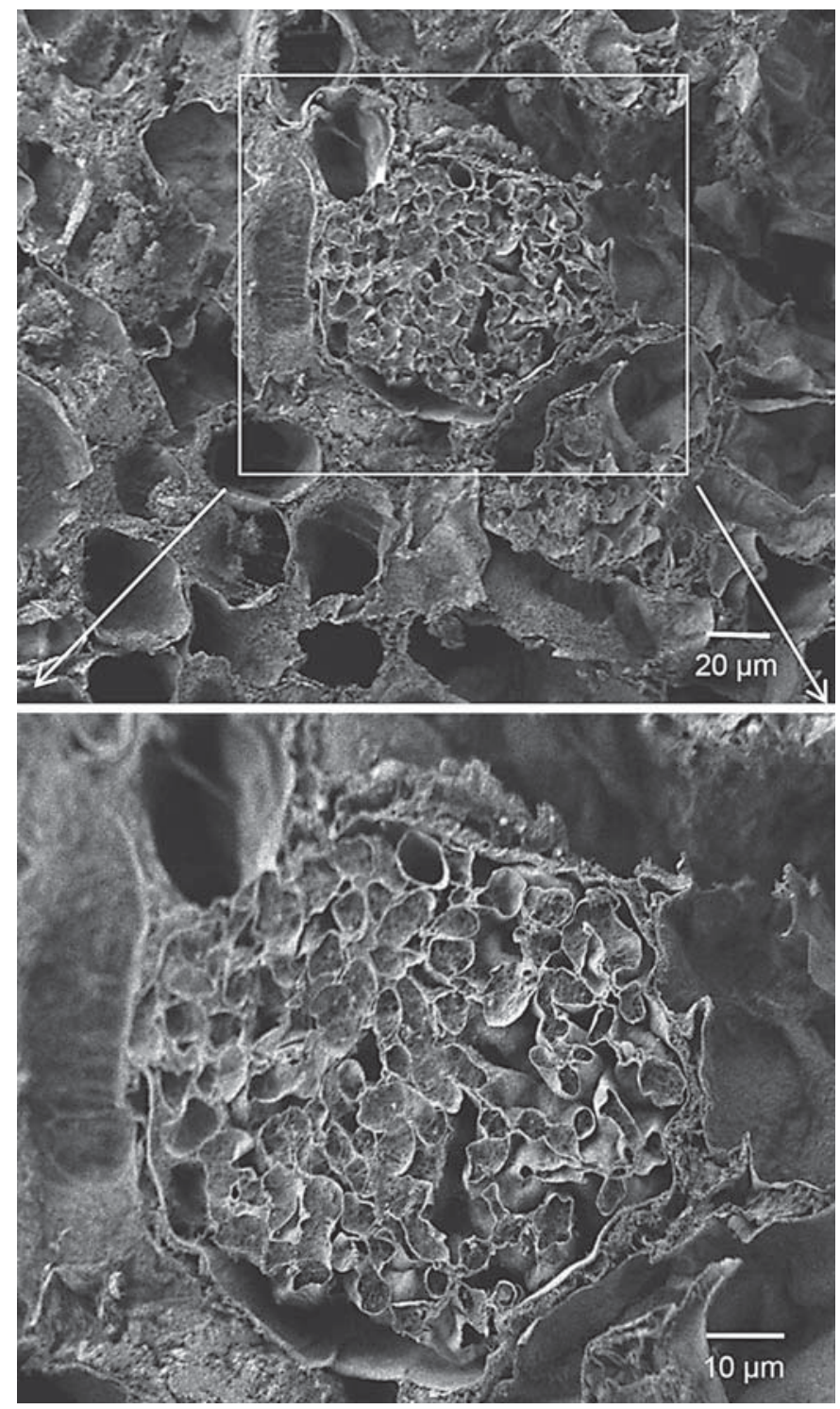

Fig. 2. Scanning electron microscopy images of transverse sections of decellularized scaffold confirm the complete removal of cellular material and maintenance of a well-organized 3D architecture of the kidney matrix.

proteins displayed expression patterns in decellularized tissue similar to those in native tissue.

In summary, the recent progress in the development of whole-organ decellularization techniques has allowed the production of biological kidney scaffolds composed of functional and structural ECM components. Detailed investigations using scanning and transmission electron microscopy have shown that these scaffolds are characterized by intact arterial and venous vasculatures, as well as by microcirculation, at the glomerular and peritubular capillaries. Besides the structure, also the composition of the acellular ECM has been experimentally investigated. Most important ECM proteins are present in these acellular matrices (e.g. collagen, laminin, fibronectin, and proteoglycans) [17]. In general, the demonstration that kidney acellular scaffolds are similar to the ECM of native kidneys in terms of their structure and composition is the basis for further development of protocols for recellularization of these matrices with the aim of reconstructing different kidney cell components.

\section{State of the Art in the Recellularization of Acellular Kidney Scaffolds}

In order to regenerate cellular compartments within an acellular scaffold, 2 main compartments must be repopulated, i.e. the kidney parenchyma to allow organspecific function, and the endothelial layer to allow perfusion of the implanted graft with the blood of the recipient. The decellularized ECM scaffold structure is considered critical to directing the cell seeding and reconstitution of specific cellular compartments [18]. The persistence of the intact glomerular, tubular, and vascular structures within the renal ECM scaffold, as well as native chemical or physical signals, could facilitate the recognition by precursor cells of their natural niche along the nephron and the vascular segments during the recellularization process. Given the complexity of the kidney structure, which includes over 30 different cell types, stem or progenitor cells are necessary to regenerate kidneys for transplantation.

The first attempt at recellularization of kidney scaffolds was reported in 2009 by Ross et al. [10]. They manually infused murine embryonic stem cells via the renal artery and the ureter. That preliminary study showed that infused cells repopulated mainly arterial vessels and glomerular capillaries. They also demonstrated differentiation of the infused cells with a loss of the embryonic phenotype and the start of the expression of markers of endothelial and epithelial differentiation [10]. In similar studies, we perfused murine embryonic stem cells to recellularize rat kidney scaffolds via the renal artery (fig. 1c), maintaining controlled pressure conditions to allow a more adequate oxygen delivery into the kidney scaffold and to avoid cell apoptosis. With this approach, we obtained a uniform distribution of seeded murine embryonic stem cells in the glomerular microvasculature, with some cells reaching the peritubular capillaries. 
Based on these studies, it is evident that cell seeding within the kidney tubules and peritubular capillaries is very difficult to achieve. Even cell infusion through the ureter cannot efficiently deliver cellular material because of the geometry of the renal tubules that are closed conduits. Very recently, a new modality of recellularization was reported [19] as an approach to possibly overcome this limitation. This new protocol consists of perfusion of a decellularized rat kidney with differentiated endothelial cells through the renal artery, followed by perfusion of the neonatal kidney cells via the ureter, maintaining a negative pressure (about $40 \mathrm{~mm} \mathrm{Hg}$ ) outside the kidney during cell perfusion. Despite these sophisticated perfusion conditions, after maturation in a bioreactor, the cells in the epithelial and endothelial compartments were mostly confined to the glomerular level, without reaching the renal tubules and venous circulation. Despite this limited extent of cell seeding, the authors claimed that repopulated kidneys were able to exert some excretory function during in vitro perfusion, as measured by a small degree of fluid excretion through the ureter and a low value of creatinine clearance. These results are difficult to explain, considering that cellularization of the tubular compartment was almost absent in this study. In addition, a certain amount of fluid and creatine excretion is expected to be present simply as a result of creatinine filtration across the decellularized matrix at the glomerular and tubular levels, rather than effective fluid filtration at the glomerular level and tubular reabsorption.

\section{Conclusions and Future Directions}

Altogether, these results suggest that we are still in the early stages of research aimed at repopulating acellular kidney scaffolds and that the processes tested so far need to be greatly improved. The achievement of this important goal may likely depend on the use of pluripotent stem cells in combination with more favorable pressure conditions to obtain a uniform flow distribution along the complex structure of the kidney matrix. The experimental studies reported so far on the development of bioengineered kidneys are probably still in the early stages, but they have certainly aroused interest and have yielded important preliminary results. We are still far from having established techniques to recreate complex organs in the laboratory, but important progress has been made in producing acellular matrices.

Recellularization of the scaffolds is definitely the most complex phase. Normally the organs are composed of ful-
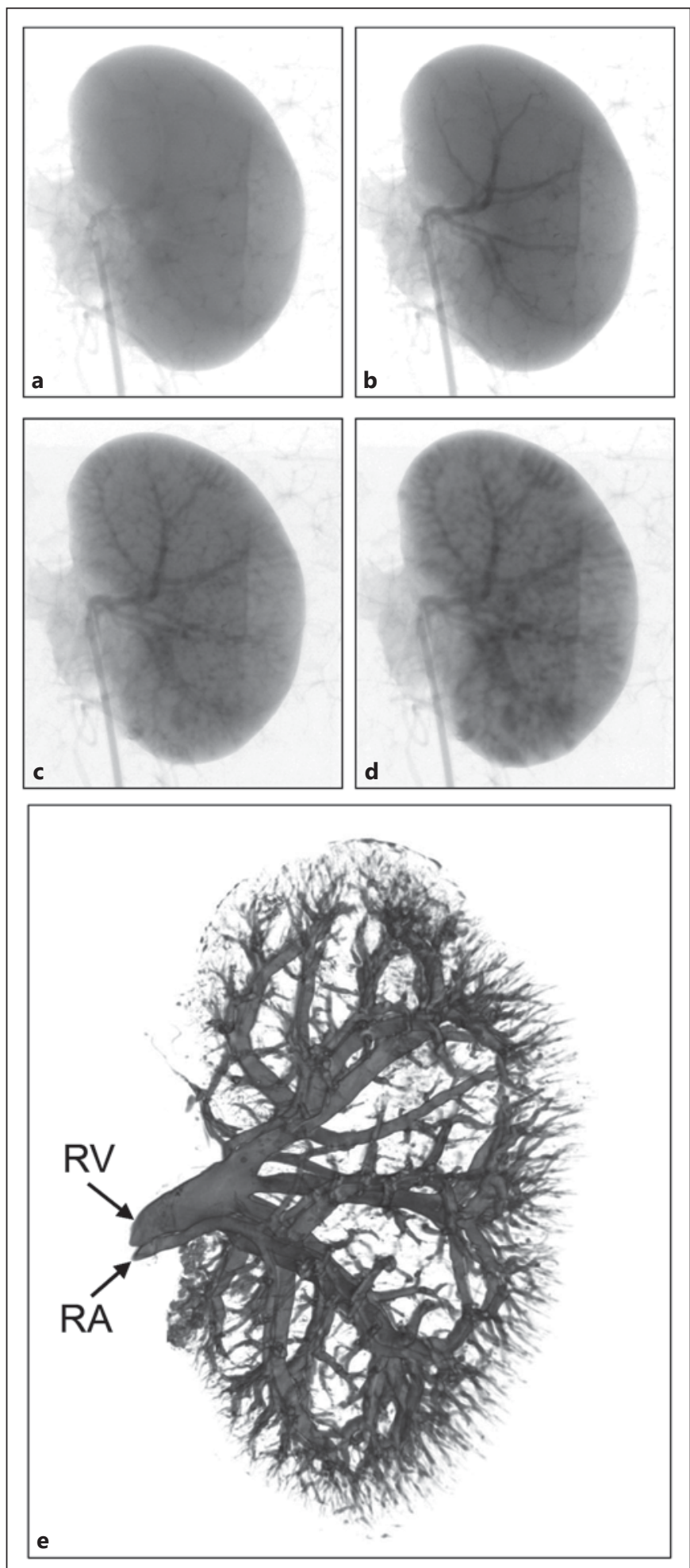

Fig. 3. Micro-CT sequence of a representative rat kidney acellular scaffold during infusion of iohexol solution (a-d) and 3D digital reconstruction of the scaffold after Microfil infusion (e) demonstrating a uniform connection of the renal artery (RA) to the renal vein $(\mathrm{RV})$ through the capillary bed. 
ly differentiated cell populations, although the presence of niches of stem or pluripotent cells has been demonstrated. However, no study has yielded sufficient results in terms of renal scaffold repopulation with an adequate number of cells, with appropriate differentiation, and with the necessary heterogeneity to attempt organ transplantation. Normally, epithelial layers divide the luminal compartment of the ducts from the vascular microcirculation, and it is therefore difficult to deliver cells to both compartments via simple perfusion through the renal artery. It may be possible that, using more complex perfusion protocols, some progress will be achieved soon. Based on the development thus far, we conclude that renal tissue engineering is in its very early stages. While it is evident that production in the laboratory of new kidneys ready for transplantation will not be ready soon, this line of research may help to discover new features of kidney cell biology, cell differentiation, and remodeling that may be useful as new evidence regarding the mechanisms of kidney pathophysiology.

\section{Acknowledgments}

The authors thank Dr. Barbara Bonandrini for her excellent assistance in the experimental investigations. This study was supported in part by the RESET project (No. 268632) of the European Research Council (FP7/2007-2013).

\section{References}

1 Lozano R, Naghavi M, Foreman K, et al: Glob$\mathrm{al}$ and regional mortality from 235 causes of death for 20 age groups in 1990 and 2010: a systematic analysis for the Global Burden of Disease Study 2010. Lancet 2012;380:20952128.

2 Couser WG, Remuzzi G, Mendis S, Tonelli M: The contribution of chronic kidney disease to the global burden of major noncommunicable diseases. Kidney Int 2011;80:1258-1270.

- 3 Wolfe RA, Ashby VB, Milford EL, Ojo AO, Ettenger RE, Agodoa LY, Held PJ, Port FK: Comparison of mortality in all patients on dialysis, patients on dialysis awaiting transplantation, and recipients of a first cadaveric transplant. N Engl J Med 1999;341:17251730.

4 Orlando G, Wood KJ, Stratta RJ, Yoo JJ, Atala A, Soker S: Regenerative medicine and organ transplantation: past, present, and future. Transplantation 2011;91:1310-1317.

5 Brown B, Lindberg K, Reing J, Stolz DB, Badylak SF: The basement membrane component of biologic scaffolds derived from extracellular matrix. Tissue Eng 2006;12:519-526.

6 Gilbert TW, Stewart-Akers AM, SimmonsByrd A, Badylak SF: Degradation and remodeling of small intestinal submucosa in canine Achilles tendon repair. J Bone Joint Surg Am 2007;89:621-630
7 Badylak S, Meurling S, Chen M, Spievack A, Simmons-Byrd A: Resorbable bioscaffold for esophageal repair in a dog model. J Pediatr Surg 2000;35:1097-1103.

-8 Gong J, Sagiv O, Cai H, Tsang SH, Del Priore LV: Effects of extracellular matrix and neighboring cells on induction of human embryonic stem cells into retinal or retinal pigment epithelial progenitors. Exp Eye Res 2008;86: 957-965.

-9 Song JJ, Ott HC: Organ engineering based on decellularized matrix scaffolds. Trends $\mathrm{Mol}$ Med 2011;17:424-432.

10 Ross EA, Williams MJ, Hamazaki T, Terada N, Clapp WL, Adin C, Ellison GW, Jorgensen M, Batich CD: Embryonic stem cells proliferate and differentiate when seeded into kidney scaffolds. J Am Soc Nephrol 2009;20:23382347.

11 Liu CX, Liu SR, Xu AB, Kang YZ, Zheng SB, Li HL: Preparation of whole-kidney acellular matrix in rats by perfusion (in Chinese). Nan Fang Yi Ke Da Xue Xue Bao 2009;29:979-982.

$\checkmark 12$ Baptista PM, Orlando G, Mirmalek-Sani SH, Siddiqui M, Atala A, Soker S: Whole organ decellularization - a tool for bioscaffold fabrication and organ bioengineering. Conf Proc IEEE Eng Med Biol Soc 2009;2009:65266529.

13 Sullivan DC, Mirmalek-Sani SH, Deegan DB, Baptista PM, Aboushwareb T, Atala A, Yoo JJ: Decellularization methods of porcine kidneys for whole organ engineering using a highthroughput system. Biomaterials 2012;33: 7756-7764.
14 Woods T, Gratzer PF: Effectiveness of three extraction techniques in the development of a decellularized bone-anterior cruciate ligament-bone graft. Biomaterials 2005;26:73397349.

15 Cartmell JS, Dunn MG: Development of cellseeded patellar tendon allografts for anterior cruciate ligament reconstruction. Tissue Eng 2004;10:1065-1075.

-16 Nakayama KH, Batchelder CA, Lee CI, Tarantal AF: Decellularized rhesus monkey kidney as a three-dimensional scaffold for renal tissue engineering. Tissue Eng Part A 2010;16:2207-2216.

17 Badylak SF, Freytes DO, Gilbert TW: Extracellular matrix as a biological scaffold material: structure and function. Acta Biomater 2009;5:1-13.

18 Badylak SF, Taylor D, Uygun K: Whole-organ tissue engineering: decellularization and recellularization of three-dimensional matrix scaffolds. Annu Rev Biomed Eng 2011;13:2753.

19 Song JJ, Guyette JP, Gilpin SE, Gonzalez G, Vacanti JP, Ott HC: Regeneration and experimental orthotopic transplantation of a bioengineered kidney. Nat Med 2013;19:646651 